\title{
Monotone method for nonlinear second order periodic boundary value problems with Carathéodory functions
}

\author{
by Ming-Xing Wang (Beijing), \\ Alberto Cabada (Santiago de Compostela) \\ and Juan J. Nieto (Santiago de Compostela)
}

\begin{abstract}
The purpose of this paper is to study the periodic boundary value problem $-u^{\prime \prime}(t)=f\left(t, u(t), u^{\prime}(t)\right), u(0)=u(2 \pi), u^{\prime}(0)=u^{\prime}(2 \pi)$ when $f$ satisfies the Carathéodory conditions. We show that a generalized upper and lower solution method is still valid, and develop a monotone iterative technique for finding minimal and maximal solutions.
\end{abstract}

1. Introduction. In this paper we consider the following periodic boundary value problem (PBVP for short) of second order:

$$
\text { (P) }\left\{\begin{array}{l}
-u^{\prime \prime}(t)=f\left(t, u(t), u^{\prime}(t)\right) \\
u(0)=u(2 \pi), u^{\prime}(0)=u^{\prime}(2 \pi) .
\end{array}\right.
$$

As is well known, the method of upper and lower solutions has been successfully applied to study this PBVP when $f$ is a continuous function (see $[2-6,12]$ and the monograph $[9]$ and the references therein).

Here, we generalize the method of upper and lower solutions to the case when $f$ is a Carathéodory function. We point out that for $f$ continuous the classical arguments of $[2-6,9,12]$ are no longer valid since the solutions are in the Sobolev space $W^{2,1}(I), I=[0,2 \pi]$. Thus, if $u$ is a solution, $u^{\prime \prime}$ is not necessarily continuous on $I$ but only $u^{\prime \prime} \in L^{1}(I)$.

Our ideas are in the spirit of $[7,10]$ where $f\left(t, u(t), u^{\prime}(t)\right)=f(t, u(t))$. There, when $u$ is bounded we deduce that $u^{\prime \prime}$ is bounded, and so is $u^{\prime}$. In our situation, we have to find a bound for the derivative of a solution since the

1991 Mathematics Subject Classification: Primary 34B15, 34C25.

Key words and phrases: upper and lower solutions, monotone iterative technique, Carathéodory function.

Research of the second and third authors partially supported by DGCYT, projects PS88-0054 and PB91-0793, and by Xunta de Galicia, project XUGA 20701A90. 
derivative of the modified problem relative to $(\mathrm{P})$ may be unbounded. To this purpose we prove a new result (Theorem 1). Thus, we improve the results of [8] where we require $f$ to be locally Lipschitzian or locally equicontinuous in some variables. The proof of some known results are included for the convenience of the reader: For instance, Lemma 4 is taken from [10]. Also we note that part (c) of Lemma 1 is proved in [4] and Theorem 2 is related to the results of Adje in [1] but our proof is simpler using a convenient modified problem.

When $v$ and $w$ are (generalized) lower and upper solutions relative to (P) and $v \leq w$, we denote by $S[v, w]$ the set of solutions of $(\mathrm{P})$ in the sector $[v, w]=\left\{u \in W^{2,1}(I): v(t) \leq u(t) \leq w(t)\right.$ for $\left.t \in I\right\}$ (see [7, 10]). We generalize the monotone method [9] to obtain minimal and maximal solutions as limits of monotone iterates.

2. The method of upper and lower solutions. We shall suppose that $f: I \times \mathbb{R}^{2} \rightarrow \mathbb{R}, I=[0,2 \pi]$, is a Carathéodory function, that is:

(i) for a.e. $t \in I$, the function $(u, s) \in \mathbb{R}^{2} \rightarrow f(t, u, s) \in \mathbb{R}$ is continuous,

(ii) for every $(u, s) \in \mathbb{R}^{2}$, the function $t \in I \rightarrow f(t, u, s)$ is measurable,

(iii) for every $R>0$, there exists a real-valued function $h(t)=h_{R}(t) \in$ $L^{1}(I)$ such that

$$
|f(t, u, s)| \leq h(t)
$$

for a.e. $t \in I$ and every $(u, s) \in \mathbb{R}^{2}$ satisfying $|u| \leq R,|s| \leq R$.

A function $u \in W^{2,1}(I)$ is a solution of $(\mathrm{P})$ if (1.1) holds for a.e. $t \in I$, and $u$ satisfies (1.2). When $f$ is continuous, any solution of $(\mathrm{P})$ is a classical solution, that is, a $C^{2}$-solution. If, in addition, $f$ is $2 \pi$-periodic in $t$, then any solution can be extended by periodicity to $\mathbb{R}$, and then it is a periodic solution of (1.1).

Let us say that a function $v: I \rightarrow \mathbb{R}$ is a lower solution of $(\mathrm{P})$ if $v \in W^{2,1}(I)$,

$$
-v^{\prime \prime}(t) \leq f\left(t, v(t), v^{\prime}(t)\right) \quad \text { for a.e. } t \in I
$$

and

$$
v(0)=v(2 \pi), \quad v^{\prime}(0) \geq v^{\prime}(2 \pi) .
$$

Similarly, $w: I \rightarrow \mathbb{R}$ is an upper solution of $(\mathrm{P})$ if $w \in W^{2,1}(I)$,

$$
-w^{\prime \prime}(t) \geq f\left(t, w(t), w^{\prime}(t)\right) \quad \text { for a.e. } t \in I
$$

and

$$
w(0)=w(2 \pi), \quad w^{\prime}(0) \leq w^{\prime}(2 \pi) .
$$

Throughout we shall suppose that $v \leq w$ on $I$. We shall consider the following condition: 
(H1) There exists $g: \mathbb{R}^{+} \rightarrow \mathbb{R}^{+}$continuous such that

$$
|f(t, u, s)| \leq g(|s|)
$$

for a.e. $t \in I$ with $v(t) \leq u \leq w(t)$, and $s \in \mathbb{R}$, satisfying

$$
\int_{\lambda}^{\infty} \frac{s}{g(s)+C} d s=\infty \quad \forall \lambda>0 \text { and } \forall C>0 .
$$

Note that the usual Nagumo condition $\int_{\lambda}^{\infty}(s / g(s)) d s=\infty$ implies (2.6) when either $\limsup _{s \rightarrow \infty} g(s)<\infty$ or $\liminf _{s \rightarrow \infty} g(s)>0$.

Now, we give a priori estimates for the derivative of solutions of $(\mathrm{P})$.

LEMMA 1. Let $0 \leq t_{1}<t_{2} \leq 2 \pi, u \in W^{2,1}\left(\left[t_{1}, t_{2}\right]\right)$ and assume that $v \leq u \leq w$ on $\left[t_{1}, t_{2}\right]$ and (1.1) is satisfied for a.e. $t \in\left[t_{1}, t_{2}\right]$. If (H1) holds, then there exists a positive constant $N$ which depends only on $v, w, g$ and a constant $C$, such that:

(a) $u^{\prime}\left(t_{1}\right) \leq C$ or $u^{\prime}\left(t_{2}\right) \leq C$ implies $u^{\prime}(t) \leq N$ on $\left[t_{1}, t_{2}\right]$.

(b) $u^{\prime}\left(t_{1}\right) \geq C$ or $u^{\prime}\left(t_{2}\right) \geq C$ implies $u^{\prime}(t) \geq-N$ on $\left[t_{1}, t_{2}\right]$.

(c) $u\left(t_{1}\right)-u\left(t_{2}\right)=u^{\prime}\left(t_{1}\right)-u^{\prime}\left(t_{2}\right)=0$ implies $\left|u^{\prime}(t)\right| \leq N$ on $\left[t_{1}, t_{2}\right]$.

Proof. (a) Suppose that $u^{\prime}\left(t_{1}\right) \leq C$ and that

$$
\forall n \in \mathbb{N}, \exists T_{n} \in\left[t_{1}, t_{2}\right] \text { such that } u^{\prime}\left(T_{n}\right)=n .
$$

Let $n_{0} \in \mathbb{N}$ be such that

$$
\int_{|C|}^{n_{0}} \frac{s}{g(s)} d s>\max _{t \in I} w(t)-\min _{t \in I} v(t) .
$$

By (2.7) there exists $\bar{t} \in\left[t_{1}, T_{n_{0}}\right]$ such that $u^{\prime}(\bar{t})=|C|$ and $0 \leq|C| \leq$ $u^{\prime}(t) \leq n_{0}$ for all $t \in\left[\bar{t}, T_{n_{0}}\right]$. In this interval we obtain

$$
\left|-u^{\prime \prime}(t)\right|=\left|f\left(t, u(t), u^{\prime}(t)\right)\right| \leq g\left(\left|u^{\prime}(t)\right|\right)
$$

and

Thus,

$$
\frac{u^{\prime \prime} u^{\prime}}{g\left(u^{\prime}\right)} \leq \frac{\left|-u^{\prime \prime}\right| u^{\prime}}{g\left(\left|u^{\prime}\right|\right)} \leq u^{\prime}
$$

$$
\begin{aligned}
\int_{\bar{t}}^{T_{n_{0}}} \frac{u^{\prime}(t) u^{\prime \prime}(t)}{g\left(u^{\prime}(t)\right)} d t & \leq \int_{\bar{t}}^{T_{n_{0}}} u^{\prime}(t) d t=u\left(T_{n_{0}}\right)-u(\bar{t}) \\
& \leq w\left(T_{n_{0}}\right)-v(\bar{t}) \leq \max _{t \in I} w(t)-\min _{t \in I} v(t) .
\end{aligned}
$$

On the other hand,

$$
\int_{\bar{t}}^{T_{n_{0}}} \frac{u^{\prime}(t) u^{\prime \prime}(t)}{g\left(u^{\prime}(t)\right)} d t=\int_{|C|}^{n_{0}} \frac{s}{g(s)} d s>\max _{t \in I} w(t)-\min _{t \in I} v(t) .
$$


As a consequence, there exists $N>0$ such that $u^{\prime}(t) \leq N$ on $\left[t_{1}, t_{2}\right]$.

If $u^{\prime}\left(t_{2}\right) \leq C$ and the assertion of (a) is not satisfied, then we deduce that property $(2.7)$ holds.

Let $n_{1} \in \mathbb{N}$ be such that

$$
\int_{|C|}^{n_{1}} \frac{s}{g(s)} d s>\max _{t \in I} w(t)-\min _{t \in I} v(t) .
$$

By (2.7) there exists $T \in\left[T_{n_{1}}, t_{2}\right]$ such that $u^{\prime}(T)=|C|$ and $0 \leq|C| \leq$ $u^{\prime}(t) \leq n_{1}$ for all $t \in\left[T_{n_{1}}, T\right]$. Thus,

$$
-\frac{u^{\prime \prime} u^{\prime}}{g\left(u^{\prime}\right)} \leq \frac{\left|-u^{\prime \prime}\right| u^{\prime}}{g\left(\left|u^{\prime}\right|\right)} \leq u^{\prime} \quad \text { on }\left[T_{n_{1}}, T\right]
$$

and

$$
-\int_{T_{n_{1}}}^{T} \frac{u^{\prime}(t) u^{\prime \prime}(t)}{g\left(u^{\prime}(t)\right)} d t \leq \int_{T_{n_{1}}}^{T} u^{\prime}(t) d t \leq \max _{t \in I} w(t)-\min _{t \in I} v(t) .
$$

On the other hand,

$$
-\int_{T_{n_{1}}}^{T} \frac{u^{\prime}(t) u^{\prime \prime}(t)}{g\left(u^{\prime}(t)\right)} d t=-\int_{n_{1}}^{|C|} \frac{s}{g(s)} d s=\int_{|C|}^{n_{1}} \frac{s}{g(s)} d s>\max _{t \in I} w(t)-\min _{t \in I} v(t) .
$$

Therefore there exists $N>0$ such that $u^{\prime}(t) \leq N$ on $\left[t_{1}, t_{2}\right]$.

Analogously we prove (b). The proof of (c) is given in Lemma 3.2 of $[4]$.

For any $u \in X=C^{1}(I)$, we define

$$
p(t, u)= \begin{cases}v(t), & u<v(t) \\ u, & v(t) \leq u \leq w(t) \\ w(t), & u>w(t)\end{cases}
$$

We obtain the following series of results:

Lemma 2. For $u \in X$, the following two properties hold:

(a) $\frac{d}{d t} p(t, u(t))$ exists for a.e. $t \in I$.

(b) If $u, u_{m} \in X$ and $u_{m} \stackrel{X}{\longrightarrow} u$, then

$$
\left\{\frac{d}{d t} p\left(t, u_{m}(t)\right)\right\} \rightarrow \frac{d}{d t} p(t, u(t)) \quad \text { for a.e. } t \in I .
$$

Proof. Note that if $u \in X$ then $u^{+}=\max \{u, 0\}$ and $u^{-}=\max \{-u, 0\}$ are absolutely continuous. We rewrite $p(t, u)=[u-v(t)]^{-}-[u-w(t)]^{+}+u$. Because $u, v, w \in X$, it is enough to prove that if $u, u_{m} \in X$ and $u_{m} \stackrel{X}{\longrightarrow} u$, 
then

$$
\left\{\frac{d}{d t} p\left(t, u_{m}^{ \pm}(t)\right)\right\} \rightarrow \frac{d}{d t} p\left(t, u^{ \pm}(t)\right) \quad \text { for a.e. } t \in I .
$$

Since $\frac{d}{d t} u_{m}^{+}(t), \frac{d}{d t} u^{+}(t)$ exist for a.e. $t \in I$, suppose that $t_{0} \in I$ is such that $\frac{d}{d t} u_{m}^{+}\left(t_{0}\right)$ and $\frac{d}{d t} u^{+}\left(t_{0}\right)$ exist for all $m=1,2, \ldots$

If $u\left(t_{0}\right)>0$, then $u\left(t_{0}\right)=u^{+}\left(t_{0}\right)>0$. Therefore $\frac{d}{d t} u^{+}\left(t_{0}\right)=\frac{d}{d t} u\left(t_{0}\right)$ and there exists $M \in \mathbb{N}$ such that $u_{m}\left(t_{0}\right)=u_{m}^{+}\left(t_{0}\right)>0$ for all $m \geq M$. Thus

$$
\frac{d}{d t} u_{m}^{+}\left(t_{0}\right)=\frac{d}{d t} u_{m}\left(t_{0}\right) \rightarrow \frac{d}{d t} u\left(t_{0}\right) .
$$

If $u\left(t_{0}\right)<0$, then there exists $M>0$ such that $u_{m}\left(t_{0}\right)<0$ for all $m \geq M$. Therefore $u_{m}(t)<0$ on $\left(t_{0}-\delta_{m}, t_{0}+\delta_{m}\right)$ for some $\delta_{m}>0$ and then $u_{m}^{+}(t)=0$ on $\left(t_{0}-\delta_{m}, t_{0}+\delta_{m}\right)$. Hence $\frac{d}{d t} u_{m}^{+}\left(t_{0}\right)=\frac{d}{d t} u^{+}\left(t_{0}\right)=0$ and then obviously

$$
\frac{d}{d t} u_{m}^{+}\left(t_{0}\right) \rightarrow \frac{d}{d t} u^{+}\left(t_{0}\right) \quad \text { as } m \rightarrow \infty .
$$

If $u\left(t_{0}\right)=0$, then $u^{+}\left(t_{0}\right)=0$. Since $\frac{d}{d t} u^{+}\left(t_{0}\right)$ exists, we have $\frac{d}{d t} u^{+}\left(t_{0}\right)$ $=0$. It is easy to prove that $\frac{d}{d t} u\left(t_{0}\right)=0$.

Because $\frac{d}{d t} u_{m}^{+}\left(t_{0}\right)$ exists, we find that

$$
\frac{d}{d t} u_{m}^{+}\left(t_{0}\right)= \begin{cases}u_{m}^{\prime}\left(t_{0}\right), & u_{m}\left(t_{0}\right)>0 \\ 0, & u_{m}\left(t_{0}\right) \leq 0\end{cases}
$$

Therefore

$$
\left|\frac{d}{d t} u_{m}^{+}\left(t_{0}\right)\right| \leq\left|\frac{d}{d t} u_{m}\left(t_{0}\right)\right| \rightarrow\left|\frac{d}{d t} u\left(t_{0}\right)\right|=0=\frac{d}{d t} u^{+}\left(t_{0}\right) .
$$

Similarly, we can prove the conclusions about $u^{-}(t)$, and thus the proof of Lemma 2 is complete.

Now, consider the following modified problem:

$$
\left\{\begin{array}{l}
-u^{\prime \prime}+u=f^{*}\left(t, u, u^{\prime}\right)+p(t, u) \\
u(0)=u(2 \pi), u^{\prime}(0)=u^{\prime}(2 \pi)
\end{array}\right.
$$

where $f^{*}\left(t, u(t), u^{\prime}(t)\right)=f\left(t, p(t, u(t)), \frac{d}{d t} p(t, u(t))\right)$.

Since $u \in X, \frac{d}{d t} p(t, u(t))$ exists for a.e. $t \in I$. If $t_{0} \in I$ is such that $\frac{d}{d t} p\left(t_{0}, u\left(t_{0}\right)\right)$ does not exist, then it is easy to prove that the left and right derivatives of $p(t, u(t))$ at $t_{0}$ must exist and both values depend only on the $X$-norms of $u, v$ and $w$. Therefore we can complement the values of $\frac{d}{d t} p(t, u(t))$ in such a way that it is bounded and the bound depends only on the $X$-norm of $u, v$ and $w$. For any $z \in X$, the linear problem

$$
\left\{\begin{array}{l}
-u^{\prime \prime}+u=f^{*}\left(t, z(t), z^{\prime}(t)\right)+p(t, z(t)) \equiv \sigma(t) \\
u(0)=u(2 \pi), u^{\prime}(0)=u^{\prime}(2 \pi)
\end{array}\right.
$$


has a unique solution $u$ given by the formula

$$
u(t)=C_{1} e^{t}+C_{2} e^{-t}-\frac{e^{t}}{2} \int_{0}^{t} \sigma(s) e^{-s} d s+\frac{e^{-t}}{2} \int_{0}^{t} \sigma(s) e^{s} d s
$$

where

$$
\begin{aligned}
C_{1} & =\frac{1}{2\left(e^{2 \pi}-1\right)} \int_{0}^{2 \pi} \sigma(s) e^{2 \pi-s} d s, \\
C_{2} & =\frac{1}{2\left(e^{2 \pi}-1\right)} \int_{0}^{2 \pi} \sigma(s) e^{s} d s .
\end{aligned}
$$

Note that $\sigma(t)=f^{*}\left(t, z(t), z^{\prime}(t)\right)+p(t, z(t))$ is measurable, $|p(t, z(t))| \leq$ $R$ and $\left|\frac{d}{d t} p(t, z(t))\right| \leq R$, which implies that $\left|f^{*}\left(t, z(t), z^{\prime}(t)\right)\right| \leq h(t) \in L^{1}(I)$ and $\sigma \in L^{1}(I)$.

From (2.10) and the formula

$$
u^{\prime}(t)=C_{1} e^{t}-C_{2} e^{-t}-\frac{e^{t}}{2} \int_{0}^{t} \sigma(s) e^{-s} d s+\frac{e^{-t}}{2} \int_{0}^{t} \sigma(s) e^{s} d s
$$

it is clear that $u \in X$.

Define the operator $T: X \rightarrow X$, where $T(z)=u$, with $u$ defined by (2.10). For this operator we obtain the following result

Lemma 3. $T: X \rightarrow X$ is compact.

Proof. Let $z_{m} \in X, m \in \mathbb{N}, z_{m} \stackrel{X}{\longrightarrow} z, T\left(z_{m}\right)=u_{m}, T(z)=u$. We have $\left\|z_{m}\right\|_{X} \leq M$ for some $M>0$. Then $p\left(t, z_{m}(t)\right) \rightarrow p(t, z(t))$ for a.e. $t \in I$ and $\left|p\left(t, z_{m}(t)\right)\right| \leq M,\left|\frac{d}{d t} p\left(t, z_{m}(t)\right)\right| \leq N$ for a.e. $t \in I$ and for some $N$ depending only on $M, v$ and $w$.

Now, $\left|f^{*}\left(t, z(t), z^{\prime}(t)\right)\right| \leq h(t) \in L^{1}(I)$ follows from (2.1). By the hypothesis on $f$ and Lemma 2 we know that $f^{*}\left(t, z_{m}(t), z_{m}^{\prime}(t)\right)$ converges to $f^{*}\left(t, z(t), z^{\prime}(t)\right)$ in measure. Hence, by the Lebesgue dominated convergence theorem,

$$
\lim _{m \rightarrow \infty} \int_{0}^{t} \sigma_{m}(s) e^{ \pm s} d s=\int_{0}^{t} \sigma(s) e^{ \pm s} d s
$$

where $\sigma_{m}(t)=f^{*}\left(t, z_{m}(t), z_{m}^{\prime}(t)\right)+p\left(t, z_{m}(t)\right)$.

By $(2.10)$ and $(2.11)$ we have $\lim _{m \rightarrow \infty}\left(u_{m}(t), u_{m}^{\prime}(t)\right)=\left(u(t), u^{\prime}(t)\right)$ for every $t \in I$. Because $\left|\sigma_{m}(s)\right| \leq h(s)+|v(s)|+|w(s)| \in L^{1}(I)$, the sequence $\left\{g_{m}(t)\right\}=\left\{\int_{0}^{t} \sigma_{m}(s) e^{ \pm s} d s\right\}$ is equicontinuous, and so are $\left\{u_{m}(t)\right\}$ and $\left\{u_{m}^{\prime}(t)\right\}$.

It is obvious that $\left\{u_{m}(t), u_{m}^{\prime}(t)\right\}$ is uniformly bounded. Therefore, by the Ascoli theorem $u_{m} \stackrel{X}{\longrightarrow} u$. Hence $T$ is continuous. 
Similarly, for any bounded set $B \subset X$, let $B_{1}=\{u: u=T(z)$ for some $z \in B\}$ and $B_{2}=\left\{u^{\prime}: u \in B_{1}\right\}$. Then $B_{1}$ and $B_{2}$ are equicontinuous and uniformly bounded. Thus, there exist subsequences $\left\{u_{m}\right\}=\left\{T z_{m}\right\} \subset B_{1}$ and $\left\{u_{m}^{\prime}\right\} \subset B_{2}$ such that $u_{m} \rightarrow u$ and $u_{m}^{\prime} \rightarrow \bar{u}$ uniformly on $I$. Using (2.10) and (2.11) it is easy to prove that $\bar{u}(t)=u^{\prime}(t)$. In consequence, $u_{m} \stackrel{X}{\longrightarrow} u$, and this shows that $T$ is compact.

LEMMA 4. Let $y \in W^{2,1}(I)$ and suppose that there exists $M \in L^{1}(I)$ such that $M(t)>0$ for a.e. $t \in I$ and $y^{\prime \prime}(t) \geq M(t) y(t)$ for a.e. $t \in I$, $y(0)=y(2 \pi), y^{\prime}(0) \geq y^{\prime}(2 \pi)$. Then $y(t) \leq 0$ for every $t \in I$.

Proof. The proof can be found in [10, Lemma 3.1] and we present it for the sake of completeness. If $X \subset I$ is such that $y(t)>0$ for a.e. $t \in X$, then $y^{\prime \prime}(t)>0$ for a.e. $t \in X$. In consequence, there exists at least one $\tau \in I$ with $y(\tau) \leq 0$. If $y(0)>0$, then there exist $0 \leq s_{1} \leq s_{2} \leq 2 \pi$ with $y\left(s_{1}\right)=y\left(s_{2}\right)=0$ and $y(s)>0$ for $s \in J=\left[0, s_{1}\right) \cup\left(s_{2}, 2 \pi\right] \subset X$. Thus, $y^{\prime}$ is nondecreasing on $J$ and we get a contradiction since $y^{\prime}(0) \geq y^{\prime}(2 \pi)$. Hence, $y(0) \leq 0$.

Now, if $\max \{y(s): s \in I\}=y\left(t_{0}\right)>0$, then there exist $t_{1}, t_{2} \in(0,2 \pi)$ such that $t_{1}<t_{0}<t_{2}, y\left(t_{1}\right)=y\left(t_{2}\right)=0$, and $y(s)>0$ for $s \in\left(t_{1}, t_{2}\right)$. In consequence, $y^{\prime}$ is nondecreasing on $\left(t_{1}, t_{2}\right)$, and this is not possible since $y\left(t_{1}\right)=y\left(t_{2}\right)=0$ and $y\left(t_{0}\right)>0$.

LEMMA 5. Let $u \in W^{2,1}\left(\left[t_{1}, t_{2}\right]\right), h \in L^{1}\left(\left[t_{1}, t_{2}\right]\right)$ and $c$ be constant, $-u^{\prime \prime}(t)=f(t)$, with $|f(t)| \leq h(t)$ for a.e. $t \in\left[t_{1}, t_{2}\right]$. Then there exists a constant $N>0$ depending only on $c$ and $h$ such that:

(a) $u^{\prime}\left(t_{1}\right) \leq c$ or $u^{\prime}\left(t_{2}\right) \leq c$ implies $u^{\prime}(t) \leq N$ on $\left[t_{1}, t_{2}\right]$.

(b) $u^{\prime}\left(t_{1}\right) \geq c$ or $u^{\prime}\left(t_{2}\right) \geq c$ implies $u^{\prime}(t) \geq-N$ on $\left[t_{1}, t_{2}\right]$.

Proof. (a) If $u^{\prime}\left(t_{1}\right) \leq c$, taking into account that $u^{\prime \prime}(t) \leq\left|-u^{\prime \prime}(t)\right|=$ $|f(t)| \leq h(t)$, we obtain

$$
u^{\prime}(t) \leq u^{\prime}\left(t_{1}\right)+\int_{t_{1}}^{t} h(s) d s \leq c+\|h\|_{1} \quad \text { on }\left[t_{1}, t_{2}\right] .
$$

If $u^{\prime}\left(t_{2}\right) \leq c$, then from $-u^{\prime \prime}(t) \leq\left|-u^{\prime \prime}(t)\right|=|f(t)| \leq h(t)$ we get

$$
u^{\prime}(t) \leq u^{\prime}\left(t_{2}\right)+\int_{t_{2}}^{t} h(s) d s \leq c+\|h\|_{1} \quad \text { on }\left[t_{1}, t_{2}\right] .
$$

(b) If $u^{\prime}\left(t_{1}\right) \geq c$, then $-u^{\prime}(t) \leq-u^{\prime}\left(t_{1}\right)+\|h\|_{1} \leq-c+\|h\|_{1}$ on $\left[t_{1}, t_{2}\right]$, that is, $u^{\prime}(t) \geq-N$ on $\left[t_{1}, t_{2}\right]$.

If $u^{\prime}\left(t_{2}\right) \geq c$, then $-u^{\prime}(t) \leq-u^{\prime}\left(t_{2}\right)+\|h\|_{1} \leq-c+\|h\|_{1}$, i.e. $u^{\prime}(t) \geq-N$ on $\left[t_{1}, t_{2}\right]$. 
Using the previous lemmas we obtain the following a priori estimate for the solutions of problem (2.8).

THEOREM 1. There exists a constant $M>0$ such that if $\lambda \in[0,1], u \in X$ and $u=\lambda T u$, then $\|u\|_{X} \leq M$.

Proof. The equation $u=\lambda T u$ is equivalent to

$$
\left\{\begin{array}{l}
-u^{\prime \prime}+u=\lambda f^{*}\left(t, u(t), u^{\prime}(t)\right)+\lambda p(t, u(t)) \\
u(0)=u(2 \pi), u^{\prime}(0)=u^{\prime}(2 \pi)
\end{array}\right.
$$

We divide the proof into two parts:

Step 1: Estimate for $u(t)$. Let $I^{0}=(0,2 \pi), A_{1}=\left\{t \in I^{0}: u(t)>w(t)\right\}$. We distinguish two cases:

(1.a) $I^{0}=A_{1}$. Then, for a.e. $t \in I^{0}$ we have

$$
-u^{\prime \prime}(t)+u(t)=\lambda f\left(t, w(t), w^{\prime}(t)\right)+\lambda w(t) \leq-\lambda w^{\prime \prime}(t)+\lambda w(t) .
$$

Hence, $y(t)=u(t)-\lambda w(t)$ satisfies

$$
\left\{\begin{array}{l}
y^{\prime \prime}(t) \geq y(t) \quad \text { for a.e. } t \in I^{0} \\
y(0)=y(2 \pi), y^{\prime}(0) \geq y^{\prime}(2 \pi) .
\end{array}\right.
$$

From Lemma 4 we conclude that $y \leq 0$, that is, $u \leq \lambda w \leq C$ on $I$.

(1.b) $I^{0} \neq A_{1}$. Thus, there exists $s_{1} \in I^{0}$ such that $u\left(s_{1}\right) \leq w\left(s_{1}\right)$. We first prove that there exists a positive constant $C$ depending only on $w$ such that $u(0) \leq C$. Obviously this is true if $u(0) \leq w(0)$.

In case $u(0)>w(0)$, let $y(t)=u(t)-\lambda w(t)$. We suppose that $y(0)>0$ since $y(0) \leq 0$ implies that $u(0) \leq \lambda w(0)$

For $y^{\prime}(0) \geq 0$, let $t_{0}=\sup \{t \in I: y(s)>0$ for $s \in[0, t)\}$ and

$$
t^{*}=\sup \left\{t \in\left[0, s_{1}\right): u(s)>w(s) \text { for } s \in[0, t)\right\} .
$$

Then $t^{*} \leq s_{1}<2 \pi, u\left(t^{*}\right)=w\left(t^{*}\right)$ and $u>w$ on $\left[0, t^{*}\right)$.

We shall prove that $t_{0}>t^{*}$. If not, $y^{\prime \prime}(t) \geq y(t)>0$ for a.e. $t \in\left[0, t_{0}\right)$ and $y^{\prime}(t)>y^{\prime}(0) \geq 0$. Hence, $y^{\prime}\left(t_{0}\right)>y^{\prime}(0)>0$. By the definition of $t_{0}$ we see that $t_{0}=2 \pi$, and $y^{\prime}(2 \pi)>y^{\prime}(0)$. This implies that $w^{\prime}(2 \pi)<w^{\prime}(0)$, a contradiction with (2.5). This shows that $t_{0}>t^{*}$.

Therefore $y^{\prime \prime}(t) \geq y(t)>0$ for a.e. $t \in\left[0, t^{*}\right)$ and thus $y^{\prime}(t)>y^{\prime}(0) \geq 0$. This implies that $y(0) \leq y\left(t^{*}\right)=u\left(t^{*}\right)-\lambda w\left(t^{*}\right)=(1-\lambda) w\left(t^{*}\right)$ and that $u(0) \leq \lambda w(0)+(1-\lambda) w\left(t^{*}\right) \leq C$.

For $y^{\prime}(0)<0$, we have $y^{\prime}(2 \pi) \leq y^{\prime}(0)<0, y(2 \pi)=y(0)>0$, $u(2 \pi)-w(2 \pi)=u(0)-w(0)>0$. Choosing $t_{1}=\inf \{t \in I: y(s)>0$ for $s \in(t, 2 \pi]\}$ and $\bar{t}=\inf \left\{t \in\left(s_{1}, 2 \pi\right]: u(s)>w(s)\right.$ for $\left.s \in(t, 2 \pi]\right\}$ and reasoning as in the previous case we again obtain $u(0) \leq C$.

We decompose $A_{1}=\bigcup\left(a_{i}, b_{i}\right)$ so that $u(t)>w(t)$ for $t \in\left(a_{i}, b_{i}\right)$ and

$$
-y^{\prime \prime}(t)+y(t) \leq 0 \quad \text { for a.e. } t \in\left(a_{i}, b_{i}\right) \text {. }
$$


By the definition of $a_{i}$ and $b_{i}$ we obtain $y\left(a_{i}\right)=(1-\lambda) w\left(a_{i}\right)$ and $y\left(b_{i}\right)=$ $(1-\lambda) w\left(b_{i}\right)$. In consequence, there exists $C \in \mathbb{R}$ such that

$$
y\left(a_{i}\right) \leq C \text { and } y\left(b_{i}\right) \leq C .
$$

Now, (2.13) and (2.14) imply that $y(t) \leq C+1$ for $t \in\left(a_{i}, b_{i}\right)$. Therefore, $u(t) \leq C+1+\lambda w(t) \leq M$ on $\bar{A}_{1}$. Obviously, $u \leq M$ on $I \backslash \bar{A}_{1}$ and thus $u \leq M$ on $I$.

Similarly, we can prove that $u \geq-M$ on $I$. Hence $|u(t)| \leq M$ for any $t \in I$.

Step 2: Estimate for $u^{\prime}(t)$. Let $B=\{t \in I: v(t)<u(t)<w(t)\}$. Suppose that $B \neq \emptyset$. Then $p(t, u(t))=u(t)$ for $t \in B$ and $u(t) \leq v(t)$ or $u(t) \geq w(t)$ for $t \in I \backslash B$. We write $B=\bigcup\left(a_{i}, b_{i}\right)$ since $B$ is an open set. For $\left(a_{i}, b_{i}\right)$, only one of the following situations hold:

(2.i) $0<a_{i}<b_{i}<2 \pi,\left[u\left(a_{i}\right)-v\left(a_{i}\right)\right] \cdot\left[w\left(a_{i}\right)-u\left(a_{i}\right)\right]=0,\left[u\left(b_{i}\right)-v\left(b_{i}\right)\right] \cdot$ $\left[w\left(b_{i}\right)-u\left(b_{i}\right)\right]=0$ and $v(t)<u(t)<w(t)$ for $t \in\left(a_{i}, b_{i}\right)$.

(2.ii) $a_{i}=0$ or $b_{i}=2 \pi$.

In the first situation we have $p(t, u(t))=u(t)$ and $\frac{d}{d t} p(t, u(t))=u^{\prime}(t)$.

Now, consider the following four cases:

(2.i.I) $u\left(a_{i}\right)=v\left(a_{i}\right)$ and $u\left(b_{i}\right)=v\left(b_{i}\right)$. Then $u^{\prime}\left(a_{i}\right) \geq v^{\prime}\left(a_{i}\right)$ and $u^{\prime}\left(b_{i}\right) \leq$ $v^{\prime}\left(b_{i}\right)$. Thus,

$$
\begin{aligned}
-u^{\prime \prime}=\lambda f\left(t, u, u^{\prime}\right)+(\lambda-1) u & \equiv \widetilde{f}\left(t, u, u^{\prime}\right), \\
\left|\widetilde{f}\left(t, u, u^{\prime}\right)\right| \leq g\left(\left|u^{\prime}\right|\right)+C & \equiv \widetilde{g}\left(\left|u^{\prime}\right|\right)
\end{aligned}
$$

and, by the hypothesis (H1),

$$
\int_{\lambda}^{\infty} \frac{s}{\tilde{g}(s)+K} d s=\infty \quad \forall \lambda>0 \text { and } \forall K>0 .
$$

By Lemma 1 we know that there exists a constant $N$ depending only on $g, v$ and $w$ such that $\left|u^{\prime}\right| \leq N$ on $\left[a_{i}, b_{i}\right]$.

(2.i.II) $u\left(a_{i}\right)=w\left(a_{i}\right)$ and $u\left(b_{i}\right)=w\left(b_{i}\right)$. Then $\left|u^{\prime}\right| \leq N$ on $\left[a_{i}, b_{i}\right]$.

(2.i.III) $u\left(a_{i}\right)=v\left(a_{i}\right)$ and $u\left(b_{i}\right)=w\left(b_{i}\right)$. Then $u^{\prime}\left(a_{i}\right) \geq v^{\prime}\left(a_{i}\right)$ and $u^{\prime}\left(b_{i}\right) \geq w^{\prime}\left(b_{i}\right)$. By Lemma $1, u^{\prime}(t) \geq-N$ on $\left[a_{i}, b_{i}\right]$.

If $u^{\prime}\left(a_{i}\right)=v^{\prime}\left(a_{i}\right)$ or $u^{\prime}\left(b_{i}\right)=w^{\prime}\left(b_{i}\right)$, then by Lemma $1, u^{\prime} \leq N$ on $\left[a_{i}, b_{i}\right]$. Otherwise $u^{\prime}\left(a_{i}\right)>v^{\prime}\left(a_{i}\right)$ and $u^{\prime}\left(b_{i}\right)>w^{\prime}\left(b_{i}\right)$. Let $a=\inf \left\{t: u^{\prime}(s)>v^{\prime}(s)\right.$ for $\left.s \in\left(t, a_{i}\right)\right\}$ and $b=\sup \left\{t: u^{\prime}(s)>w^{\prime}(s)\right.$ for $\left.s \in\left(b_{i}, t\right)\right\}$. Then $a<a_{i}<$ $b_{i}<b, u^{\prime}(a) \geq v^{\prime}(a)$ and $u^{\prime}(b) \geq w^{\prime}(b)$. Moreover, $u^{\prime}>v^{\prime}$ on $\left(a, a_{i}\right)$ and $u^{\prime}>w^{\prime}$ on $\left(b_{i}, b\right)$.

Now, $u\left(a_{i}\right)=v\left(a_{i}\right)$ and $u\left(b_{i}\right)=w\left(b_{i}\right)$ imply that $u>w$ on $\left(b_{i}, b\right]$ and $u<v$ on $\left[a, a_{i}\right)$. We conclude that $\left(u^{\prime}(a)-v^{\prime}(a)\right) \cdot\left(u^{\prime}(b)-w^{\prime}(b)\right)=0$. Otherwise, $u^{\prime}(a)>v^{\prime}(a)$ and $u^{\prime}(b)>w^{\prime}(b)$. Therefore $a=0$ and $b=2 \pi$ by 
the definitions of $a$ and $b$. Thus $u(0)<v(0) \leq w(0)=w(2 \pi)<u(2 \pi)$, and this is a contradiction.

If $u^{\prime}(b)=w^{\prime}(b)$, then $-u^{\prime \prime}=\lambda f\left(t, w, w^{\prime}\right)+\lambda w-u \leq \lambda h(t)+c$ for a.e. $t \in\left(b_{i}, b\right]$. By integration,

$$
\begin{aligned}
u^{\prime}(t) & \leq \lambda \int_{t}^{b} h(s) d s+2 \pi c+u^{\prime}(b) \\
& =w^{\prime}(b)+\lambda \int_{t}^{b} h(s) d s+2 \pi c \leq C \quad \text { on }\left(b_{i}, b\right] .
\end{aligned}
$$

Hence $u^{\prime}\left(b_{i}\right) \leq C$. Using again Lemma 1 we have $u^{\prime}(t) \leq N$ on $\left[a_{i}, b_{i}\right]$. If $u^{\prime}(a)=v^{\prime}(a)$, then similarly we see that $u^{\prime}(t) \leq N$ on $\left[a_{i}, b_{i}\right]$. Hence $\left|u^{\prime}\right| \leq N$ on $\left[a_{i}, b_{i}\right]$.

(2.i.IV) If $u\left(b_{i}\right)=v\left(b_{i}\right)$ and $u\left(a_{i}\right)=w\left(a_{i}\right)$, then analogously to (2.i.III), $\left|u^{\prime}\right| \leq N$ on $\left[a_{i}, b_{i}\right]$.

To show (2.ii), suppose $a_{i}=0$; the boundary conditions for $v, u$ and $w$ imply that $b_{i}=2 \pi$.

Let $a=\sup \{t \in I: v(s)<u(s)<w(s)$ for $s \in[0, t)\}$. Then $u(a)=v(a)$ or $u(a)=w(a)$.

If $u(a)=v(a)$, then it is clear that $u^{\prime}(a) \leq v^{\prime}(a)$. Lemma 1 implies $u^{\prime}(t) \leq N$ for a.e. $t \in[0, a]$. If $u^{\prime}(a)=v^{\prime}(a)$ we obtain $u^{\prime}(t) \geq-N$; therefore $u^{\prime}(a)<v^{\prime}(a)$.

Now, let $t_{0}=\sup \left\{t \in I: u^{\prime}(s)<v^{\prime}(s)\right.$ for $\left.s \in(a, t)\right\}$.

If $u^{\prime}\left(t_{0}\right)<v^{\prime}\left(t_{0}\right)$ we obtain $t_{0}=2 \pi$ and $u(2 \pi)<v(2 \pi)$, which is a contradiction. In consequence, $u^{\prime}\left(t_{0}\right)=v^{\prime}\left(t_{0}\right)$ and $t_{0}<2 \pi$. In the interval $\left(a, t_{0}\right)$ we have

$$
-u^{\prime \prime}=\lambda f\left(t, v, v^{\prime}\right)+\lambda v-u \geq-\lambda h+C .
$$

Thus

$$
-\int_{t}^{t_{0}} u^{\prime \prime}(s) d s \geq K
$$

and $u^{\prime}(t) \geq K+v^{\prime}\left(t_{0}\right)=K_{1}$ on $\left(a, t_{0}\right)$. By continuity $u^{\prime}(a) \geq K_{1}$, and Lemma 1 implies $\left|u^{\prime}\right| \leq N$ on $[0, a]$.

If $u(a)=w(a)$, the reasoning is analogous.

If $b_{i}=2 \pi$, we obtain $\left|u^{\prime}\right| \leq N$ on $[b, 2 \pi]$ for

$$
b=\inf \{t \in I: v(s)<u(s)<w(s) \text { for } s \in(t, 2 \pi]\} .
$$

Thus, we obtain $\left|u^{\prime}(t)\right| \leq N$ for all $t \in B \cup D$, with

$D=\left\{a_{i}, b_{i} \in(0,2 \pi)\right.$ :

either $\left(a_{i}, b_{i}\right) \in B$; or $\left[0, b_{i}\right) \in B$; or $\left.\left(a_{i}, 2 \pi\right] \in B\right\}$. 
If $B \neq I^{0}$, let $B_{1}=\{t \in I: u(t)<v(t)\}, B_{2}=\{t \in I: u(t)>w(t)\}$. Then $B_{1} \neq I$ and $B_{2} \neq I$.

First we suppose that $B_{1} \neq \emptyset$ and $B_{2} \neq \emptyset$. Decompose $B_{1}=\bigcup\left(a_{i}, b_{i}\right)$ and $B_{2}=\bigcup\left(c_{i}, d_{i}\right)$.

For $\left(a_{i}, b_{i}\right)$, we have one of the following possibilities:

(2.A) $0<a_{i}<b_{i}<2 \pi$.

(2.B) $a_{i}=0$ or $b_{i}=2 \pi$.

In the first case $u^{\prime}\left(a_{i}\right) \leq v^{\prime}\left(a_{i}\right)$ and $u^{\prime}\left(b_{i}\right) \geq v^{\prime}\left(b_{i}\right)$. Since $-u^{\prime \prime}=$ $\lambda f\left(t, v, v^{\prime}\right)+\lambda v-u$, Lemma 5 implies $\left|u^{\prime}\right| \leq N$ on $\left[a_{i}, b_{i}\right]$.

In the second situation, we first consider $a_{i}=0$. Then $u(0)<v(0)$ and $u(2 \pi)<v(2 \pi)$, that is, $b_{i}=2 \pi$. In consequence, there exists $a \in(0,2 \pi)$ such that $u(a)=v(a)$ and $u(t)<v(t)$ on $[0, a)$. Thus, without loss of generality, we can assume $u^{\prime}(a)>v^{\prime}(a)$ (otherwise, Lemma 5 implies $\left|u^{\prime}\right| \leq N$ on $[0, a]$ ).

Now, if $v(a)<w(a)$, let $b=\sup \{t \in I: v(s)<w(s)$ for $s \in[a, t)\}$. Hence, there exists $\bar{t} \leq b$ such that $u^{\prime}(t)>v^{\prime}(t)$ on $[a, \bar{t})$. Therefore $v(t)<$ $u(t)<w(t)$ on $[a, \bar{t})$, and consequently $a \in D$. Thus $\left|u^{\prime}(a)\right| \leq N$ and Lemma 5 assures that $\left|u^{\prime}\right| \leq N$ on $[0, a]$.

On the other hand, if $v(a)=w(a)$ and $v^{\prime}(a)<u^{\prime}(a)<w^{\prime}(a)$ there exists a subinterval $(a, a+\delta) \subset(0,2 \pi)$ such that $v<u<w$ on $(a, a+\delta)$; then $a \in D$ and $\left|u^{\prime}(a)\right| \leq N$. Again, Lemma 5 implies $\left|u^{\prime}\right| \leq N$ on $[0, a]$.

If $v(a)=w(a)$ and $u^{\prime}(a)=w^{\prime}(a)$, Lemma 5 implies $\left|u^{\prime}\right| \leq N$ on $[0, a]$.

Finally, if $v(a)=w(a)$ and $u^{\prime}(a)>w^{\prime}(a)$ there exists $t_{0} \in(0,2 \pi)$ such that $u>w$ on $\left(a, t_{0}\right)$ with $u^{\prime}\left(t_{0}\right)=w^{\prime}\left(t_{0}\right)$. Therefore $-u^{\prime \prime}=\lambda f\left(t, v, v^{\prime}\right)+$ $\lambda v-u$ on $\left(a, t_{0}\right)$ and $u^{\prime}(t) \leq w^{\prime}\left(t_{0}\right)+c$ for all $t \in\left(a, t_{0}\right)$. The continuity of $u^{\prime}$ and Lemma 5 imply $\left|u^{\prime}\right| \leq N$ on $[0, a]$.

If $b_{i}=2 \pi$ the proof is analogous.

For the set $B_{2}$ the reasoning is similar.

Thus, we obtain $\left|u^{\prime}(t)\right| \leq N$ for all $t \in E \cup F \equiv S$, where $E=B \cup B_{1} \cup B_{2}$ and

$$
\begin{aligned}
& F=\left\{a_{i}, b_{i} \in(0,2 \pi):\right. \\
& \text { either } \left.\left(a_{i}, b_{i}\right) \in E ; \text { or }\left[0, b_{i}\right) \in E ; \text { or }\left(a_{i}, 2 \pi\right] \in E\right\} .
\end{aligned}
$$

If $t \in I \backslash S$, then obviously either $u(t)=v(t)$ or $u(t)=w(t)$. Also there exists $\left\{x_{n}\right\} \subset F, x_{n} \neq t$ for all $n \in \mathbb{N}$, such that $t=\lim _{n \rightarrow \infty} x_{n}$ because if there exists $\delta>0$ such that $I \cap(t-\delta, t+\delta) \cap F=\emptyset$ then $t \in S$. Since $\left|u^{\prime}\left(x_{n}\right)\right| \leq N$ for all $\left\{x_{n}\right\} \subset F$ we obtain $\left|u^{\prime}(t)\right|=\left|\lim _{n \rightarrow \infty} u^{\prime}\left(x_{n}\right)\right| \leq N$ for all $t \in I \backslash S$.

This completes the proof of Theorem 1.

Theorem 2. Suppose that $v(t) \leq w(t)$ are lower and upper solutions of $(\mathrm{P})$, respectively. If $(\mathrm{H} 1)$ holds, then there exists a solution $u$ of $(\mathrm{P})$ such that $u \in[v, w]$. 
Proof. Let $X=C^{1}(I)$. By Lemma $2, \frac{d}{d t} p(t, u(t))$ exists for a.e. $t \in I$. Problem (2.8) is equivalent to the functional equation $u=T u$, with $T$ defined as in Lemma 3. By Theorem 1 we know that every solution of $u=\lambda T u$ satisfies $\|u\|_{X} \leq M$ for some constant $M>0$. In consequence, the Shaefer theorem [11] implies that there exists a solution $u$ of problem (2.8).

Finally, we prove that every solution $u$ of $(2.8)$ is such that $u \in[v, w]$, that is, $u$ is a solution in $[v, w]$ of problem $(\mathrm{P})$. Indeed, suppose that $u>w$ on $[0,2 \pi]$. Then

$$
-u^{\prime \prime}+u=f\left(t, w, w^{\prime}\right)+w \leq-w^{\prime \prime}+w .
$$

Since $(u-w)(0)=(u-w)(2 \pi)$ and $(u-w)^{\prime}(0) \geq(u-w)^{\prime}(2 \pi)$, Lemma 4 implies that $u \leq w$ on $[0,2 \pi]$, which is a contradiction. Consequently, there exists $s \in[0,2 \pi]$ such that $u(s) \leq w(s)$. If there exists $s_{1} \in[0,2 \pi]$ with $u\left(s_{1}\right)>w\left(s_{1}\right)$, and there exists $t_{1}<t_{2}$ in $(0,2 \pi)$ such that $u>w$ on $\left(t_{1}, t_{2}\right)$, with $(u-w)\left(t_{1}\right)=(u-w)\left(t_{2}\right)=0$, then in the interval $\left(t_{1}, t_{2}\right)$ we have

$$
-u^{\prime \prime}+u=f\left(t, w, w^{\prime}\right)+w \leq-w^{\prime \prime}+w .
$$

This, together with the boundary conditions, implies that $u \leq w$ on $\left(t_{1}, t_{2}\right)$, which is a contradiction.

Therefore, suppose that there exist $t_{1} \leq t_{2}$ in $(0,2 \pi)$ such that $u>w$ on $\left[0, t_{1}\right) \cup\left(t_{2}, 2 \pi\right]$ with $(u-w)\left(t_{1}\right)=(u-w)\left(t_{2}\right)=0$. In both intervals we have $(u-w)^{\prime \prime} \geq u-w>0$.

If $(u-w)^{\prime}(0) \geq 0$ then $(u-w)^{\prime}(t)>0$ for any $t \in\left[0, t_{1}\right)$ and $(u-w)\left(t_{1}\right)>$ $(u-w)(0)>0$, which is not possible.

On the other hand, if $(u-w)^{\prime}(0)<0$, we obtain $(u-w)^{\prime}(2 \pi)<0$. In consequence, $(u-w)^{\prime}<0$ on $\left(t_{2}, 2 \pi\right]$ and $(u-w)\left(t_{2}\right)>(u-w)(2 \pi)>0$.

Therefore $u \leq w$ on the interval $I$. Analogously we can prove that $u \geq v$ on $I$. Hence, every solution of (2.8) is a solution of problem (P) in the sector $[v, w]$.

This completes the proof of Theorem 2 .

3. Monotone iterative technique. Throughout this section we suppose that $v \leq w$ are lower and upper solutions of $(\mathrm{P})$, respectively. We introduce the following hypotheses:

(H2) There exists $M \in L^{1}(I)$ such that $M(t)>0$ for a.e. $t \in I$ and

$$
f(t, \phi, s)-f(t, \varphi, s) \geq-M(t)(\phi-\varphi)
$$

for a.e. $t \in I$ and every $v(t) \leq \varphi \leq \phi \leq w(t), s \in \mathbb{R}$.

There exists $N \in L^{1}(I)$ such that $N(t) \geq 0$ for a.e. $t \in I$ and

$$
f(t, u, s)-f(t, u, y) \geq-N(t)(s-y)
$$

for a.e. $t \in I$ and every $v(t) \leq u \leq w(t), s \geq y, s, y \in \mathbb{R}$. 
TheOREM 3. Suppose that (H1)-(H3) hold. Then there exist monotone sequences $v_{n} \nearrow x$ and $w_{n} \searrow z$ as $n \rightarrow \infty$, uniformly on $I$, with $v_{0}=v$ and $w_{0}=w$. Here, $x$ and $z$ are the minimal and maximal solutions of $(\mathrm{P})$ respectively on $[v, w]$, that is, if $u \in[v, w]$ is a solution of $(\mathrm{P})$, then $u \in[x, z]$. Moreover, the sequences $\left\{v_{n}\right\}$ and $\left\{w_{n}\right\}$ satisfy $v=v_{0} \leq \ldots \leq v_{n} \leq \ldots$ $\ldots \leq w_{n} \leq \ldots \leq w_{0}=w$.

Pr o of. For any $q \in[v, w] \cap X$, consider the following quasilinear periodic boundary value problem:

$$
\left\{\begin{array}{l}
-u^{\prime \prime}(t)=f\left(t, q(t), \frac{d}{d t} p(t, u(t))\right)+M(t)[q(t)-u(t)] \\
u(0)=u(2 \pi), u^{\prime}(0)=u^{\prime}(2 \pi)
\end{array}\right.
$$

Using (3.1), we deduce that if $u$ is a solution of (3.3), then

$$
\begin{aligned}
f\left(t, v(t), \frac{d}{d t} p(t, u(t))\right)+M v(t) & \leq-u^{\prime \prime}(t)+M u(t) \\
& \leq f\left(t, w(t), \frac{d}{d t} p(t, u(t))\right)+M w(t) .
\end{aligned}
$$

Using (2.1), (H1) and (3.4), and reasoning as in the proof of Theorem 1, we can say that (3.3) has a solution $u \in X$. It is not difficult (using Lemma 4) to prove that this solution is unique. Using the same arguments as in the proof of Theorem 2.1 of [10], it can be proved that $v \leq u \leq w$. Hence (3.3) is equivalent to

$$
\left\{\begin{array}{l}
-u^{\prime \prime}(t)=f\left(t, q(t), u^{\prime}(t)\right)+M(t)(q(t)-u(t)), \\
u(0)=u(2 \pi), u^{\prime}(0)=u^{\prime}(2 \pi) .
\end{array}\right.
$$

Now, define the operator $T: X \rightarrow X, T(q)=u$, where $u$ is the solution of $(3.3)$.

We shall prove that if $v \leq q_{1} \leq q_{2} \leq w, q_{1}, q_{2} \in X$, then $T\left(q_{1}\right) \leq T\left(q_{2}\right)$. Indeed, let $u_{i}=T\left(q_{i}\right), i=1,2$. Then

$$
\left\{\begin{array}{l}
-u_{i}^{\prime \prime}(t)=f\left(t, q_{i}(t), u_{i}^{\prime}(t)\right)+M(t)\left(q_{i}(t)-u_{i}(t)\right), \\
u_{i}(0)=u_{i}(2 \pi), u_{i}^{\prime}(0)=u_{i}^{\prime}(2 \pi) .
\end{array}\right.
$$

If $u_{1} \leq u_{2}$ is not true, then there exist $\varepsilon>0$ and $t_{0} \in I$ such that $u_{1}\left(t_{0}\right)=u_{2}\left(t_{0}\right)+\varepsilon$ and $u_{1} \leq u_{2}+\varepsilon$ on $I$.

First, we shall prove that there exists $\left(t_{1}, t_{2}\right) \subset I^{0}$ such that $u_{1}>u_{2}$ and $u_{1}^{\prime} \leq u_{2}^{\prime}$ on $\left(t_{1}, t_{2}\right), u_{1}^{\prime}\left(t_{1}\right)=u_{2}^{\prime}\left(t_{1}\right)$ and $u_{1}\left(t_{1}\right)-u_{2}\left(t_{1}\right) \geq u_{1}\left(t_{2}\right)-u_{2}\left(t_{2}\right)$.

Indeed, let $y(t)=u_{1}(t)-u_{2}(t)$. If there exists $\left[t_{1}, t_{2}\right]$ such that $y(t)=\varepsilon$ on $\left[t_{1}, t_{2}\right]$, then the conclusion holds. Suppose that for any subinterval $(a, b) \subset$ $I^{0}$, there exists $t \in(a, b)$ such that $y(t)<\varepsilon$. If $t_{0}=2 \pi$, then $t_{0}=0$. Thus $y(0)=y(2 \pi)=\varepsilon$ and $0 \leq y^{\prime}(2 \pi)=y^{\prime}(0) \leq 0$. If $t_{0} \in I^{0}$, then $y^{\prime}\left(t_{0}\right)=0$. Hence we always have $y^{\prime}\left(t_{0}\right)=0$. 
Since $y(0)=y(2 \pi)$, we can take $t_{0}<2 \pi$. Because $y\left(t_{0}\right)=\varepsilon \geq y(t)$ and $y(t) \not \equiv \varepsilon$ in any right neighborhood of $t_{0}$, there exists $t_{2} \in\left(t_{0}, 2 \pi\right)$ such that $y^{\prime}\left(t_{2}\right)<0$ and $y\left(t_{2}\right)>0$. Hence, there exists $t_{1} \in\left[t_{0}, t_{2}\right)$ such that $y^{\prime}\left(t_{1}\right)=0$ and $y^{\prime}(t)<0$ for $t \in\left(t_{1}, t_{2}\right]$. Consequently, $\left(t_{1}, t_{2}\right)$ satisfies our requirements.

We consider (3.6) in $\left(t_{1}, t_{2}\right)$. Since $y^{\prime} \leq 0$ on $\left(t_{1}, t_{2}\right)$, (H2) and (H3) imply that

$$
\begin{gathered}
-u_{1}^{\prime \prime}(t)+u_{2}^{\prime \prime}(t)=f\left(t, q_{1}(t), u_{1}^{\prime}(t)\right)-f\left(t, q_{2}(t), u_{2}^{\prime}(t)\right)+M(t)\left[q_{1}(t)-q_{2}(t)\right], \\
-M(t)\left[u_{1}(t)-u_{2}(t)\right] \leq-N(t)\left[u_{1}^{\prime}(t)-u_{2}^{\prime}(t)\right]-M(t)\left[u_{1}(t)-u_{2}(t)\right]
\end{gathered}
$$

for a.e. $\left(t_{1}, t_{2}\right)$.

The function $y=u_{1}-u_{2}$ satisfies

$$
\left\{\begin{array}{l}
y^{\prime \prime}(t) \geq M(t) y(t)+N(t) y^{\prime}(t)>N(t) y^{\prime}(t) \\
y\left(t_{1}\right) \geq y\left(t_{2}\right), 0=y^{\prime}\left(t_{1}\right) \geq y^{\prime}\left(t_{2}\right)
\end{array}\right.
$$

for a.e. $\left(t_{1}, t_{2}\right)$.

Solving the differential inequality, we obtain

$$
y^{\prime}\left(t_{2}\right) \exp \left(-\int_{t_{1}}^{t_{2}} N(s) d s\right)>y^{\prime}\left(t_{1}\right)=0 .
$$

This is a contradiction with $y^{\prime}\left(t_{2}\right) \leq 0$. Therefore, $u_{1} \leq u_{2}$ on $I$.

Now, define sequences $v_{0}=v, v_{n}=T\left(v_{n-1}\right), w_{0}=w$ and $w_{n}=T\left(w_{n-1}\right)$. Because the solution $u$ of (3.3) satisfies $v \leq u \leq w$ on $I$, using the monotonicity of $T$ we see that $v=v_{0} \leq v_{1} \leq \ldots \leq v_{n} \leq \ldots \leq w_{n} \leq \ldots \leq w_{1} \leq$ $w_{0}=w$. Hence, the limits $\lim _{n \rightarrow \infty} v_{n}(t)=x(t)$ and $\lim _{n \rightarrow \infty} w_{n}(t)=z(t)$ exist. Note that $v_{n}$ satisfies

$$
\left\{\begin{array}{l}
-v_{n}^{\prime \prime}(t)=f\left(t, v_{n-1}(t), v_{n}^{\prime}(t)\right)+M(t)\left[v_{n-1}(t)-v_{n}(t)\right] \equiv \widetilde{f}\left(t, v_{n}(t), v_{n}^{\prime}(t)\right), \\
v_{n}(0)=v_{n}(2 \pi), v_{n}^{\prime}(0)=v_{n}^{\prime}(2 \pi), v(t) \leq v_{n}(t) \leq w(t),
\end{array}\right.
$$

and

$$
\left|\widetilde{f}\left(t, v_{n}(t), v_{n}^{\prime}(t)\right)\right| \leq g\left(\left|v_{n}^{\prime}(t)\right|\right)+C \equiv \widetilde{g}\left(\left|v_{n}^{\prime}(t)\right|\right)
$$

and

$$
\int_{\lambda}^{\infty} \frac{s}{\widetilde{g}(s)+K} d s=\infty
$$

By Lemma 1, there exists a constant $N$ depending only on $g, v$ and $w$ such that $\left|v_{n}^{\prime}\right| \leq N$ on $I$ for any $n=1,2, \ldots$, that is, $\left\{v_{n}\right\}$ is a bounded set of $X$.

Similarly, $\left\{w_{n}\right\}$ is a bounded set of $X$. Using the same arguments as in Lemma 3 , it follows that $v_{n} \stackrel{X}{\longrightarrow} x$ and $w_{n} \stackrel{X}{\longrightarrow} z$, that is,

$\lim _{n \rightarrow \infty}\left(v_{n}(t), v_{n}^{\prime}(t), w_{n}(t), w_{n}^{\prime}(t)\right)=\left(x(t), x^{\prime}(t), z(t), z^{\prime}(t)\right) \quad$ uniformly on $I$. 
Writing the integral equations of $v_{n}$ and $w_{n}$ respectively and using standard arguments, we deduce that $x$ and $z$ satisfy $(\mathrm{P})$ and $v \leq x \leq z \leq w$ on $I$. Now, we know that if $u \in X, v \leq u \leq w$ and $u$ solves $(\mathrm{P})$, then $T u=u$, so that $v_{n} \leq u \leq w_{n}$ for any $n=1,2, \ldots$ and thus $x \leq u \leq z$ on $I$.

This completes the proof of Theorem 3 .

Acknowledgements. The authors are thankful to the referee for helpful comments and suggestions.

\section{References}

[1] A. Adje, Sur et sous-solutions généralisées et problèmes aux limites du second ordre, Bull. Soc. Math. Belgique Sér. B 42 (1990), 347-368.

[2] J. Bebernes, A simple alternative problem for finding periodic solutions of second order ordinary differential systems, Proc. Amer. Math. Soc. 42 (1974), 121-127.

[3] J. Bebernes and R. Fraker, A priori bounds for boundary sets, ibid. 29 (1971), $313-318$.

[4] J. Bebernes and W. Kelley, Some boundary value problems for generalized differential equations, SIAM J. Appl. Math. 25 (1973), 16-23.

[5] J. Bebernes and M. Martelli, On the structure of the solution set for periodic boundary value problems, Nonlinear Anal. 4 (1980), 821-830.

[6] J. Bebernes and K. Schmitt, Periodic boundary value problems for systems of second order differential equations, J. Differential Equations 13 (1973), 32-47.

[7] A. Cabada and J. J. Nieto, A generalization of the monotone iterative technique for nonlinear second-order periodic boundary value problems, J. Math. Anal. Appl. 151 (1990), 181-189.

[8] —, - Extremal solutions of second-order nonlinear periodic boundary value problems, Appl. Math. Comput. 40 (1990), 135-145.

[9] G. S. Ladde, V. Lakshmikantham and A. S. Vatsala, Monotone Iterative Techniques for Nonlinear Differential Equations, Pitman, New York, 1985.

[10] J. J. Nieto, Nonlinear second-order periodic boundary value problems with Carathéodory functions, Applicable Anal. 34 (1989), 111-128.

[11] D. R. Smart, Fixed Points Theorems, Cambridge University Press, Cambridge, 1974.

[12] M. X. Wang, Monotone method for nonlinear periodic boundary value problems, J. Beijing Inst. Technol. 9 (1989), 74-81 (in Chinese).

INSTITUTE OF SYSTEMS SCIENCE ACADEMIA SINICA BEIJING 100080, P.R. CHINA DEPARTAMENTO DE ANÁLISIS MATEMÁTICO
FACULTAD DE MATEMÁTICAS
UNIVERSIDAD DE SANTIAGO DE COMPOSTELA SANTIAGO DE COMPOSTELA, SPAIN 